\title{
Transmission dynamics and susceptibility patterns of SARS-CoV-2 in domestic, farmed and wild animals: Sustainable One health surveillance for conservation and public health to prevent future epidemics and pandemics
}

\author{
Ariful Islam¹, Jinnat Ferdous ${ }^{1}$, Shariful Islam ${ }^{1}$, Md. Abu Sayeed ${ }^{1}$, Md Rahman ${ }^{1}$, \\ Mohammad Mahmudul Hassan ${ }^{2}$, and Tahmina Shirin ${ }^{3}$ \\ ${ }^{1}$ EcoHealth Alliance \\ ${ }^{2}$ Faculty of Veterinary Medicine Chattogram Veterinary and Animal Sciences University \\ Chattogram 4225 Bangladesh \\ ${ }^{3}$ Institute of Epidemiology Disease Control and Research
}

July 14, 2021

\begin{abstract}
The exact origin of Severe Acute Respiratory Syndrome Coronavirus 2 (SARS-CoV-2) and source of introduction into humans has not been established yet, though it might be originated from animals. Therefore, we conducted a literature review to understand the putative reservoirs, transmission dynamics, and susceptibility patterns of SARS-CoV-2 in animals. Rhinolophu $s$ bats are presumed to be natural progenitors of SARS-CoV-2 related viruses. Initially pangolin was thought to be the source of spillover to human, but they might get infected from human or other animal species. So, the virus spillover pathways to humans remain unknown. Human-to-animal transmission has been testified in pet, farmed, zoo and free-ranging wild animals. Infected animals can transmit the virus to other animals in natural settings like, mink-to-mink, and mink-to-cat transmission. Animal-to-human transmission is not a persistent pathway, while mink-to-human transmission continues to be illuminated. Multiple companion and captive wild animals were infected by emerging alpha variant of concern (B.1.1.7 lineage) whereas Asiatic lions were infected by delta variant, (B.1.617.2). To date, multiple animal species- cat, ferrets, non-human primates, hamsters, and bats, showed high susceptibility to SARS-CoV-2 in experimental condition, while swine, poultry, cattle showed no susceptibility. The founding of SARS-CoV-2 in wild animal reservoirs can confronts the control of the virus in humans and might carry a risk to the welfare and conservation of wildlife as well. We suggest vaccinating pet, and captive animals to stop spillover and spillback events. We recommend sustainable one health surveillance at animal-human-environmental interface to detect and prevent future epidemics and pandemics by Disease X.
\end{abstract}

\section{Hosted file}

Main document updated.docx available at https://authorea.com/users/320785/articles/530400transmission-dynamics-and-susceptibility-patterns-of-sars-cov-2-in-domestic-farmed-andwild-animals-sustainable-one-health-surveillance-for-conservation-and-public-health-toprevent-future-epidemics-and-pandemics 